\title{
Routine Cervical Dilatation at Cesarean Section and Its Influence on Pain Scores and Post-Operative Morbidity: A Randomized Controlled Study
}

\author{
Ammar Kannawati ${ }^{1}$, Hediye Dağdeviren ${ }^{2 *}$, Ender Güven ${ }^{1}$, Ulkar \\ Heydarova $^{1}$
}

\begin{abstract}
Objective: We hypothesized that, collection of blood in the intrauterine cavity and distended uterus in women after elective caesarean section could lead to increasing pain. The aim of this study was to identify the effect of routine cervical dilation during elective caesarean section on infectious morbidity, postpartum hemorrhage, and pain after labor by using visual analogue scale (VAS).
\end{abstract}

Methods: A case-control study was conducted on 245 pregnant women who had elective cesarean section at Bakırköy Dr. Sadi Konuk Teaching and Research Hospital, Istanbul. The cases had intraoperative digital cervical dilatation at elective cesarean section while the controls did not.

Results: There was a significant reduction in hemoglobin levels postoperatively in each group ( $\mathrm{p}=0.001$ for both groups), but the level of reduction was not statistically different between the groups $(\mathrm{p}=0.37)$. The duration of operation was not statistically different between the two groups. In addition, there were no statistically significant difference in febrile morbidity $(p=0.478)$, endometritis ( $p$ $=0.311)$, wound infection $(\mathrm{p}=0.297)$ and UTI $(\mathrm{p}=0.479)$. Mean postoperative endometrial cavity thickness of the dilated group was significantly less than that of the non-dilated group $(7.8 \pm 4.1,6.8 \pm 3.8$, respectively, $\mathrm{p}=0.044)$. We found VAS scores of dilated group to be significantly less than those of the non-dilated group $(5.4 \pm 3.0,4.6 \pm 2.8$, respectively, $\mathrm{p}=0.023)$

\footnotetext{
${ }^{1}$ Sadi Konuk Training and Research Hospital, Bakirkoy, Istanbul, Turkey

2 Department of Gynecology and Obstetrics, Department of Medical Physiology, Istanbul Aydin University, Istanbul, Turkey.

* Corresponding Author

Hediye Dağdeviren hediyedagdeviren@gmail.com ORCID: 0000-0002-9384-4514

* Received: 29 May 2021 Accepted: 22 June 2021

DOI: 10.17932/EJOH.2020.022/ejoh_v02i1006
} 
Routine Cervical Dilatation at Cesarean Section and Its Influence on Pain Scores and Post-Operative Morbidity: A Randomized Controlled Study

Conclusion: In conclusion, cervical dilatation during cesarean section does not increase the incidence of postoperative endometritis or wound site infection, but it may decrease postoperative pain. However, larger studies on homogenous patient groups are required for generalization of these results.

Keywords: Cervical dilation, elective cesarean section, endometrial thickness, infectious morbidity, visual analogue score

\section{Introduction}

Cesarean section (CS), the most common obstetric surgery, can be either an elective or an emergency procedure. The surgical technique varies amongst centers and individual surgeons. Infectious morbidity is the most frequent complication of CS(1). Strategies to minimize postoperative morbidities includes modifications in surgical technique, methods of placental delivery, changing gloves and altering the uterine position during repair of the uterine incision (2-6).

A woman's cervix is firm and undilated at the beginning of pregnancy and some obstetricians consider undilated cervix at pre-labor to result in obstruction of blood and lochia drainage (7). Collection of lochia or debris may lead to postpartum hemorrhage and endometritis. To circumvent this, obstetricians routinely dilate the cervix from above. Finger, sponge forceps, or other instruments can be used for dilatation. In contrast, mechanical cervical dilatation during CS may result in cervical trauma and increase the risk of infection. Endometritis appears to result from contamination of uterus, abdominal cavity and the abdominal incision with ascending vaginal flora bacteria (8).

We hypothesized that, collection of blood in the intrauterine cavity and distended uterus in women after elective caesarean section could lead to increasing pain. Previously published studies have evaluated infectious morbidity and postpartum hemorrhage after the dilatation of the cervix during elective CS. However, none of these have evaluated effect of dilatation on pain. The aim of this study was to identify the effect of routine cervical dilation during elective caesarean section on infectious morbidity, postpartum hemorrhage, and pain after labor by using visual analogue scale (VAS) (9).

\section{Materials and Methods}

This prospective randomized study was carried out at Bakırköy Dr. Sadi Konuk Teaching and Research Hospital from January 2013 to March 2015. A total of 285 cases of elective caesarean delivery were enrolled in this study after obtaining informed consent. The Non-invasive Human Research Ethics 
Committee and Local Health Ministry Authority approved the study (Approval number: 2012-14-06, ClinicalTrials.gov Identifier: NCT01954719). Patients were randomized using computer-generated random numbers into either intraoperative cervical dilatation (Group A, $\mathrm{n}=143$ ) or no intraoperative cervical dilatation (Group $\mathrm{B}, \mathrm{n}=142$ ) groups. The investigator was not blinded to the procedure allocation. The trial profile is shown in Figure 1.

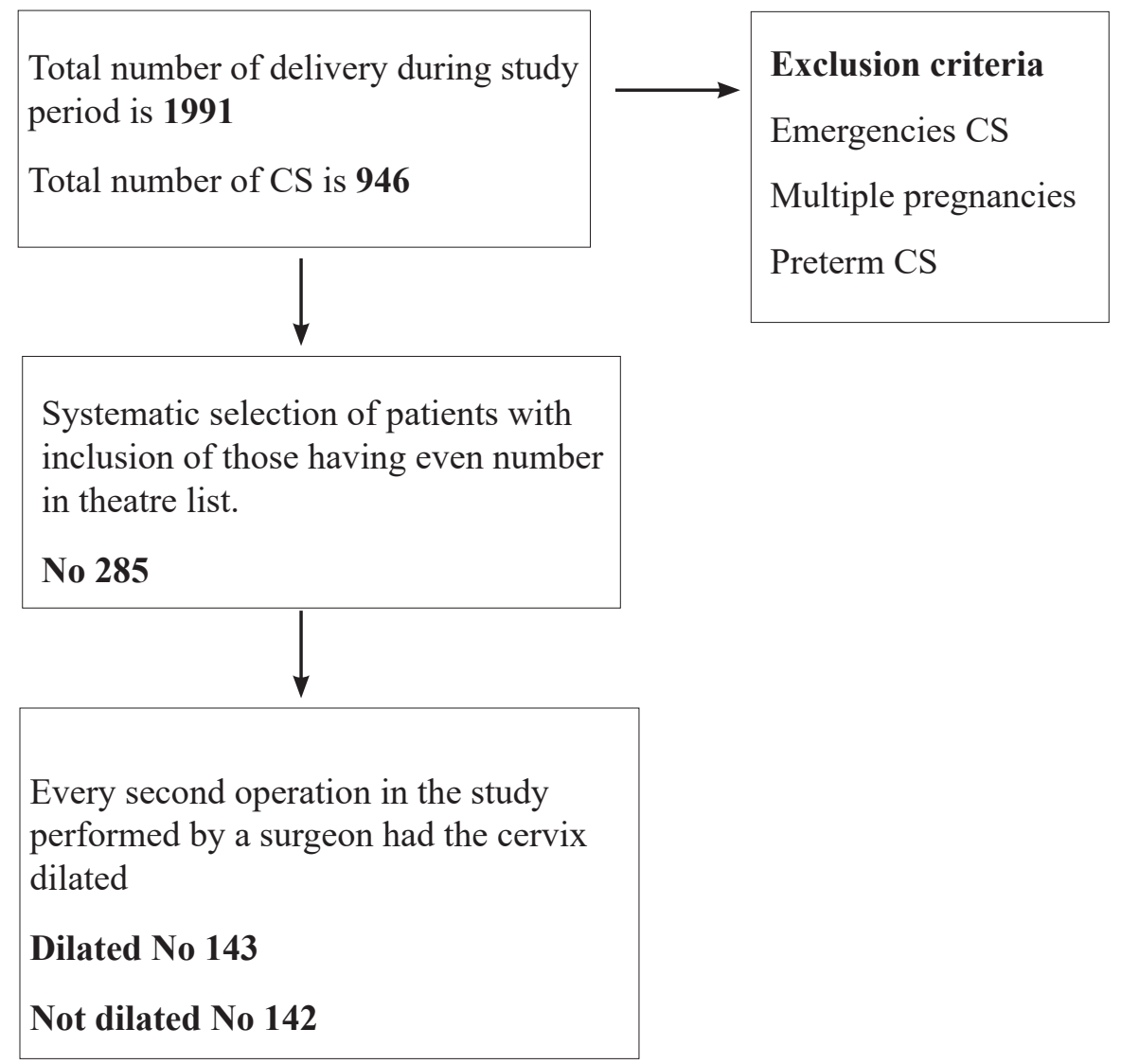

Figure 1. Consort diagram for randomization

All participants in the included studies were undergoing elective caesarean section at gestational ages more than 37 weeks. The exclusion criteria were: women undergoing CS due to multiple pregnancies, preterm births, rupture of membranes, fever on admission, chorioamnionitis, need for blood transfusion before or during caesarean section, use of antibiotics during the last $24 \mathrm{~h}$, women who felt labor pain before their previous cesarean operations. 
Routine Cervical Dilatation at Cesarean Section and Its Influence on Pain Scores and Post-Operative Morbidity: A Randomized Controlled Study

Cesarean operations were performed by the same operators. Before CS, a Foley catheter was inserted and the abdomen was cleaned with povidone iodine solution. Intravenous $1 \mathrm{~g}$ cefazolin was administered to each participant when the umbilical cord was clamped. Except for digital cervical dilatation, all surgical techniques and suture materials were same in both groups. This included pfannenstiel incision, blunt entry into the peritoneal cavity, lower segment transverse uterine incision digitally expanded to deliver the fetus a placenta. In the cervical dilatation group, the surgeon performed the cervical dilatation by inserting the double-gloved index finger into the cervical canal of the patients after the extraction of placenta and membranes. The outer glove was removed after this procedure. Suturing of the uterine incision was performed without exteriorization of the uterus. The abdominal wall was closed in two layers. Skin incisions were closed.

Postoperative patient care was the same for both groups. The temperature was measured every $4 \mathrm{~h}$ using sublingual digital devices. Clinical signs of urinary tract infection (UTI) were checked and urinalysis was performed. At $8 \mathrm{~h}$ postoperatively, urinary Foley catheters were removed and oral fluid intake was started. All patients had their complete blood count tested prior to surgery and $24 \mathrm{~h}$ postoperatively. All patients were monitored for clinical signs of post-operative infectious morbidity including febrile morbidity, wound infection, endometritis and UTI. Febrile morbidity was defined as an axillary temperature of $38^{\circ} \mathrm{C}$ or more measured twice or more after $24 \mathrm{~h}$ post-delivery. Wound infection refers to clinical evidence of purulent or serous discharge with induration, warmth and tenderness or bacteriological evidence on a swab of suspicious area of the wound. Endometritis was defined as axillary temperature greater than $38^{\circ} \mathrm{C}$ associated with purulent, foul smelling vaginal discharge or uterine tenderness on bimanual examination. UTI was suspected in the presence of symptoms and confirmatory diagnosis made when culture of midstream urine specimen revealed significant bacteriuria $(>100,000$ organisms $/ \mathrm{mL})$. All the patients were followed up with specific attention to the clinical aspects of endometritis, such as foul-smelling vaginal discharge, uterine tenderness. Wound infection was detected clinically by presence of smelly discharge, erythema, induration, and tenderness. Blood loss during CS was estimated using a drop in packed cell volume 24 hours postoperatively.

At $24 \mathrm{~h}$ post-operation, endometrial cavity thickness was measured using transabdominal ultrasound. The measurement involved both the decidual thickness and intracavitary fluid collection. Patients without infection or other complications were discharged on the third postoperative day 
Patients were informed verbally and in a written form about the timing of postpartum control examinations and the need to contact the physician in case of adverse events, such as findings of infection including fever, foul smelling vaginal discharge or abnormal bleeding after discharge from the hospital. They were invited to Bakırköy Dr. Sadi Konuk Teaching and Research Hospital Obstetrics and Gynecology clinic on the $7^{\text {th }}$ day and at 6 weeks after delivery for control examination and for detection of complications including infections in the puerperium period. On the $7^{\text {th }}$ day after operation, patients' pain levels were evaluated by using VAS (9). It is self-completed by the patients. The patients were asked to place a line to the perpendicular VAS line at the point that describing their pain intensity.

The primary outcome was rate of post-partum endometritis, postpartum hemorrhage and pain. Secondary outcomes that were analyzed included wound infection, febrile morbidity and infectious morbidity. Blood loss at surgery and operative time were compared between the two groups.

\section{Sample size estimation}

Our primary outcome was comparing of VAS scores between the two groups. Using the program 'MedCalc', we calculated that a sample size of 141 subjects would be required for each group. Influence was 0.33 with a sample size of 141 in each group that would provide $80 \%$ power to detect a $95 \%$ difference in VAS scores. Assuming a 10\% dropout rate, 128 women were required in each study group.

\section{Statistical analysis}

Data were analyzed using descriptive statistical methods (mean, standard deviation, median, frequency, rate, Minimum, Maximum). The distribution of variables was assessed by Kolmogorov-Smirnov test. Mann-Whitney U test was used for comparison of independent two groups. Wilcoxon test was used for comparison of two dependent groups. Statistical analyses were performed using Statistics Package for Social Sciences version 22.0 (SPSS Inc., Chicago, IL, USA).

\section{Results}

A total of 245 women were included in this study. Of these 245 women, 143 women had intraoperative digital cervical dilatation and 142 women with no intraoperative cervical dilatation. The mean age of patients in the cervical dilatation group and the non-dilated group was $29.8 \pm 5.4$ and $30.4 \pm 5.9$, respectively. There were no statistically significant differences between the two 
groups with regard to maternal age, gravidity, parity, gestational age on the day of cesarean, birth weight, preoperative temperature, duration of hospitalization and 1st and 5th minute Apgar scores of the babies. Furthermore, there was no statistically significant difference in the mean baseline pre-operative hemoglobin blood level between the two groups $(11.0 \pm 1.6$ vs. $11.1 \pm 1.4, \mathrm{p}=0.516)$. Please refer to Table 1 .

Table 1. Demographic and clinical properties of patients

\begin{tabular}{llll}
\hline Variable & $\begin{array}{l}\text { Cervical } \\
\text { dilatation } \\
(\mathbf{n}=\mathbf{1 4 3})\end{array}$ & $\begin{array}{l}\text { No Cervical } \\
\text { dilatation } \\
(\mathbf{n}=\mathbf{1 4 2})\end{array}$ & $\mathbf{p}$ \\
& $(\mathbf{m e a n} \pm \mathbf{S D})$ & $\mathbf{( m e a n} \pm \mathbf{S D})$ & \\
\hline Age & $29.8 \pm 5.4$ & $30.4 \pm 5.9$ & 0.364 \\
Gravidy & $2.5 \pm 1.2$ & $2.7 \pm 1.3$ & 0.083 \\
Parity & $2.0 \pm 1.1$ & $2.0 \pm 1.2$ & 0.924 \\
Gestational age at delivery & $37.4 \pm 3.0$ & $37.4 \pm 2.5$ & 0.212 \\
Birth weight $(\mathrm{kg})$ & $3001 \pm 778$ & $3078 \pm 705$ & 0.771 \\
Preoperative hemoglobin & $11.0 \pm 1.6$ & $11.1 \pm 1.4$ & 0.516 \\
blood level $(\mathrm{g} / \mathrm{L})$ & & & \\
Preoperative temperature $\left({ }^{\circ} \mathrm{C}\right)$ & $36.4 \pm 0.3$ & $36.5 \pm 0.6$ & 0.327 \\
First minute Apgar score & $7.6 \pm 1.9$ & $7.7 \pm 1.5$ & 0.524 \\
Fifth minute Apgar score & $9.1 \pm 1.5$ & $9.0 \pm 1.1$ & 0.121 \\
\hline SD: Standard deviation & & & \\
\hline
\end{tabular}

As shown in Table 2, there was a significant reduction in hemoglobin levels postoperatively in each group ( $\mathrm{p}=0.001$ for both groups), but the level of reduction was not statistically different between the groups $(p=0.37)$. In other words, preoperative and postoperative hemoglobin levels of the groups did not reveal a significant difference. The duration of operation was not statistically different between the two groups. In addition, there were no statistically significant difference in febrile morbidity $(\mathrm{p}=0.478)$, endometritis $(\mathrm{p}=0.311)$, wound infection $(\mathrm{p}=0.297)$ and UTI $(\mathrm{p}=0.479)$. Mean postoperative endometrial cavity thickness of the dilated group was significantly less than that of the non-dilated group ( $7.8 \pm 4.1,6.8 \pm 3.8$, respectively, $\mathrm{p}=0.044)$. We found the VAS scores of dilated group to be significantly less than those of the non-dilated group (5.4 \pm $3.0,4.6 \pm 2.8$, respectively, $\mathrm{p}=0.023$ ) as seen in Table2. 
Tables 2. Intra-operative and post-operative outcomes

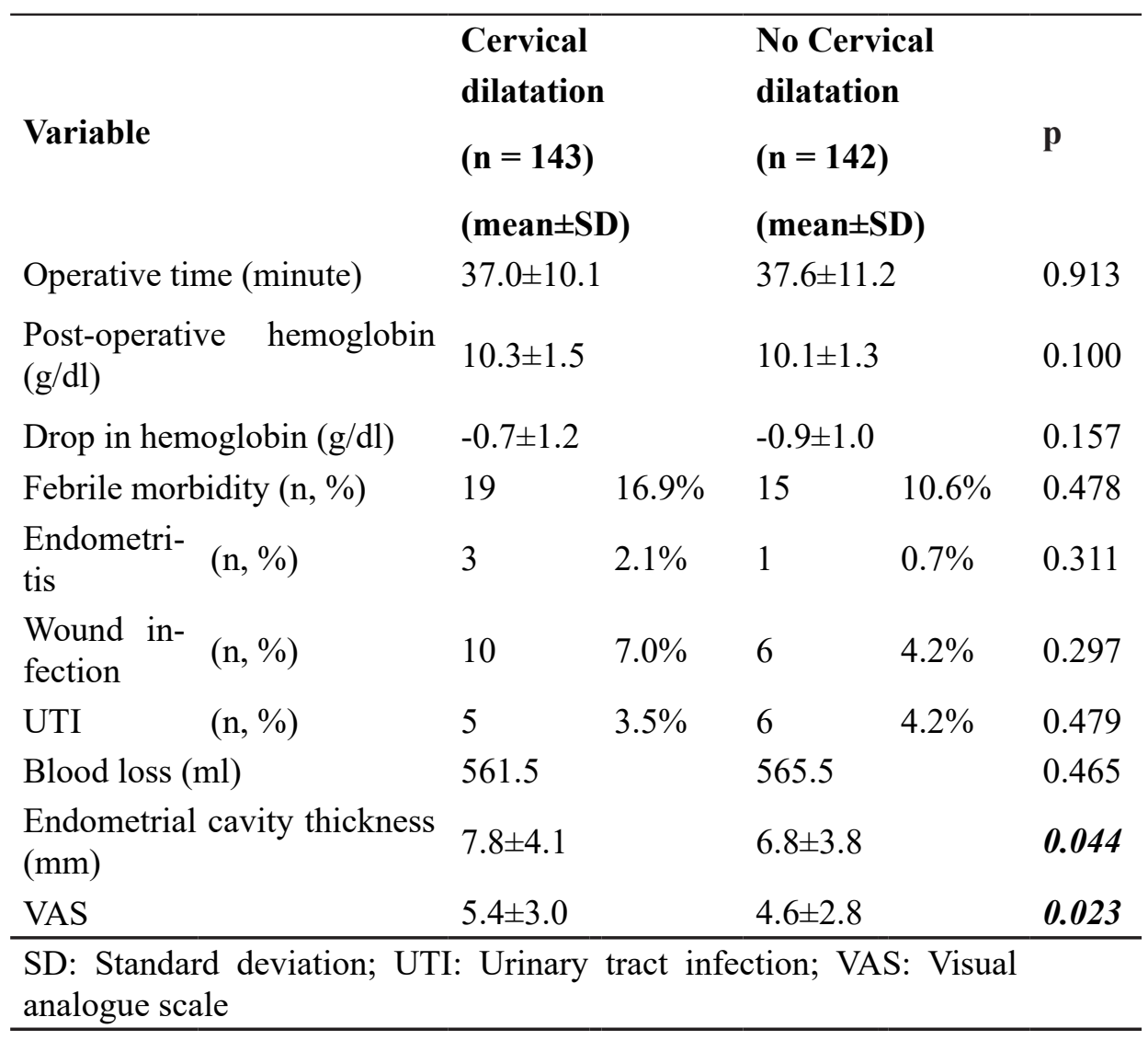

In the study population, 123 and 119 women had a previous CS in each group. The other indications for cesarean delivery in women were: Cephalopelvic disproportion $(2.1 \%, 2.8 \%)$, breech presentation $(7.7 \%, 7.7 \%)$, poor obstetric history $(1.4 \%, 2.1 \%)$, hypertensive disorders $(1.4 \%, 2.8 \%)$, placenta previa $(0.7 \%, 0.00 \%)$ and multiple gestation $(0.7 \%, 0.7 \%)$ (Table 3$)$. 
Table 3. Indications for elective cesarean sections

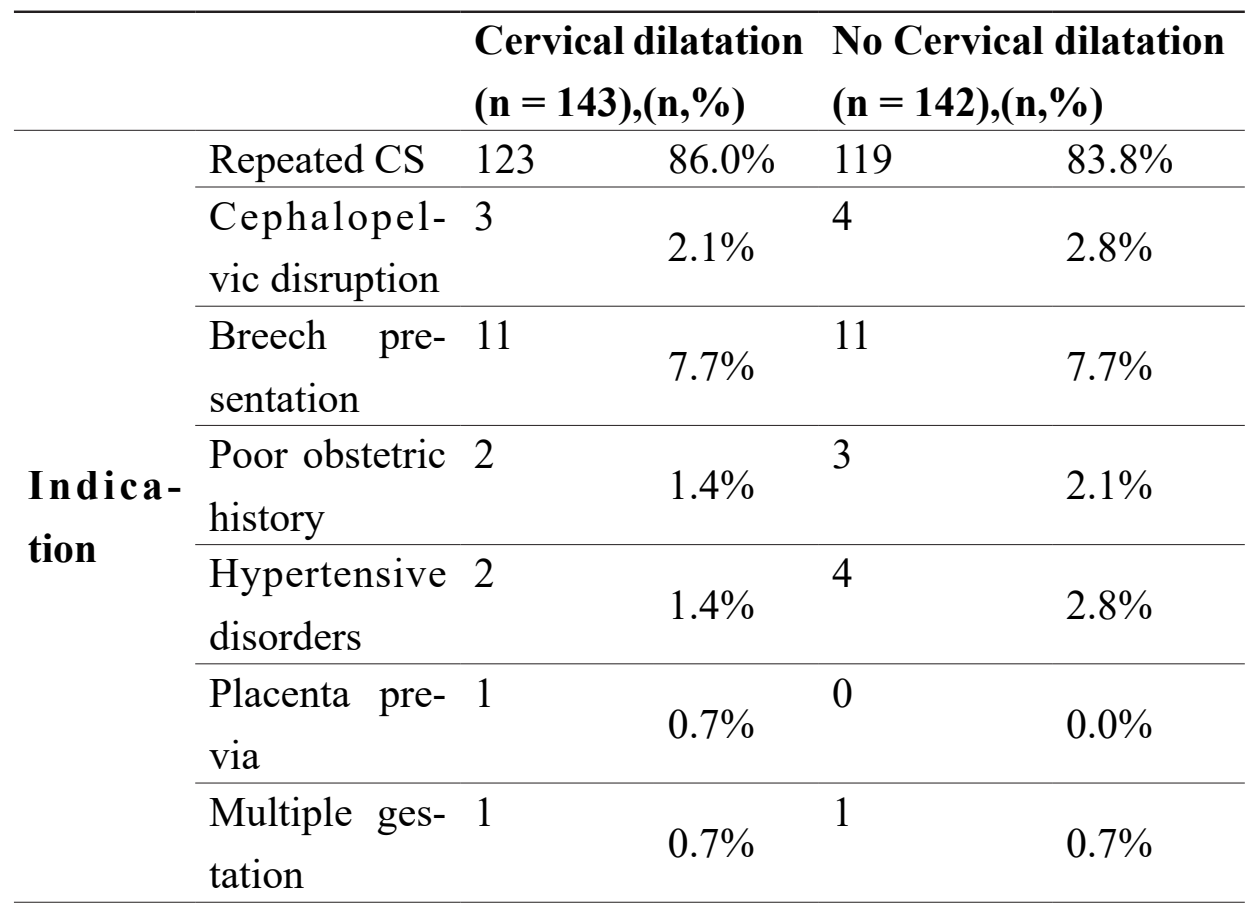

CS, caesarean section; CPD, cephalopelvic disproportion;

\section{Discussion}

Several CS methods to minimize surgery-related morbidity have been investigated. However, the advantages and disadvantages of cervical dilation during CS are still controversial. Dewhurst pointed to the pros and cons of the cervical dilation still being questionable, and thus requiring further investigation (10). The presence of dilatation during $\mathrm{C}$-section has been examined by several authors over the years and various ideas have been put forth $(11,12)$. Nevertheless, there are only a few randomized studies on the relevant topic in the literature (1316).

Bollapragada et al. emphasized the need for ultrasonography in the absence of lochia in uterine, which may be caused by intrauterine collection after $\mathrm{CS}$, and hence cervical dilatation must be performed in the patients undergoing elective CS (17). We observed in this study that the endometrial thickness was significantly reduced in the dilatation cases; however, we have doubts about the clinical significance of these results. Our study also indicates that the thickness of endometrium evaluated at postoperative $24^{\text {th }}$ hour was not associated with 
postoperative pain. There are studies suggesting an ascending bacterial infection to cause endometritis, peritonitis and wound infections in the setting of cervical dilatation $(18,19)$. However, in this study, none of the cases developed peritonitis.

In the randomized controlled study on 131 patients by Ahmet et al., no difference was detected between patients with dilation and without dilation during C-section regarding febrile morbidity and endometritis, and none of these patients developed wound site infection. Even though we obtained similar results in this study, the wound site infection was less common in patients who had dilatation, but the difference was not statistically significant. Güngördük et al. did not observe any significant difference in febrile morbidity and infection between the patients; however, they reported the duration of the operation to be significantly longer in cases with cervical dilation (15). In the present study, no difference in the duration of the operation was detected.

As a result of their study, Tosun et al. argued that routine dilation is not necessary during CS. However, researchers that have examined the endometrial thickness after cesarean section have reported the endometrium to be significantly thinner in the dilation group (13). Although similar results were seen in our study, we do not believe it to have any clinical significance. None of our patients with increased endometrial thickness and fluid accumulation in the intrauterine cavity developed a hemorrhagic complication. We did not detect any difference in VAS scores of patients with vaginal labor history; however, the heterogeneous nature of the study population is a weakness of the study.

On the other hand, the investigation of the patients' pain symptoms using the VAS on the $7^{\text {th }}$ postoperative day to identify the association of cervical dilation in CS with postoperative pain can be considered as one of the strengths of the study. We measured significantly lower VAS scores for the patients that had cervical dilation. Moreover, single center randomized study with and the performance of operations by the same surgeons are the strengths of the study.

A limitation of the current study was that the differences between the patients with normal vaginal labor history and those who had primary cesarean section could not be evaluated due to limited number of cases. Additionally, the study population was not homogeneous with regards to the number of previous CS operations. Furthermore, the influence of prior CS operation counts on VAS score could not be assessed. Nevertheless, this is one of the very few studies in the literature on the relevant topic.

In conclusion, cervical dilatation during cesarean section does not increase the incidence of postoperative endometritis or wound site infection, but it may decrease postoperative pain. However, larger studies on homogenous patient groups are required for generalization of these results. 
Routine Cervical Dilatation at Cesarean Section and Its Influence on Pain Scores and Post-Operative Morbidity: A Randomized Controlled Study

\section{References}

1. Leth RA, Møller JK, Thomsen RW, et al. Risk of selected postpartum infections after cesarean section compared with vaginal birth: a five-year cohort study of 32,468 women. Acta Obstet Gynecol Scand. 2009;88:976-983.

2. Dodd JM, Anderson ER, Gates S. Surgical techniques for uterine incision and uterine closure at the time of caesarean section. Cochrane Database Syst Rev 2008, Issue 3. Art. No: CD004732. DOI: 10.1002/14651858.CD004732. pub2.

3. Cernadas M, Smulian JC, Giannina G, Ananth CV. Effects of placental delivery method and intraoperative glove changing on postcesarean febrile morbidity. J Matern Fetal Med 1998;7:100 - 104.

4. Magann EF, Washburne JF, Harris RL, Bass JD, Duff WP, Morrison JC. Infectious morbidity, operative blood loss, and length of the operative procedure after cesarean delivery by method of placental removal and site of uterine repair. J Am Coll Surg 1995; 181:517-520.

5. Wilkinson CS, Enkin MW. Manual removal of placenta at caesarean section. Cochrane Database Syst Rev 2008, Issue 3. Art. No.: CD000130. DOI: 10.1002/14651858.CD000130.pub3.

6. Anorlu RI, Maholwana B, Hofmeyr GJ. Methods of delivering the placenta at caesarean section. Cochrane Database Syst Rev 2008, Issue 3. Art. No: CD004737. DOI: 10.1002/ 14651858.CD004737.pub2.

7. Myers KM, Paskaleva AP, House M, Socrate S. Mechanical and biochemical properties of human cervical tissue. Acta Biomaterialia 2008;4:104-16.

8. Yancey MK, Clark P, Duff P. The frequency of glove contamination during cesarean delivery. Obstet Gynecol 1994;83:538 -542.

9. Wewers M.E. \& Lowe N.K. A critical review of visual analogue scales in the measurement of clinical phenomena. Research in Nursing and Health 1990;13:227-236.

10. Johanson RB. Obstetric procedures. In: Edmonds DK, (editor). Dewhurst's Textbook of Obstetrics and Gynaecology for Postgraduates. 6 th ed. Oxford: Blackwell Science; 1999;308-29.

11. Donald I. Practical Obstetric Problems, 5th edn. London: Lloyd-Luke, 1979; 847.

12. Myerscough PR. Munro Kerr's Operative Obstetrics, 10th edn. London: Bailliere Tindall, 1982; 302.

13. Tosun M, Sakinci M, Celik H, Torumtay B, Yazici E, Alper T, et all. A 
randomized controlled study investigating the necessity of routine cervical dilatation during elective cesarean section. Arch Gynecol Obstet. 2011; 284:85-9.

14) Ahmed B, Abu Nahia F, Abushama M. Routine cervical dilatation during elective cesarean section and its influence on maternal morbidity: a randomized controlled study. J Perinat Med.2005; 33:510-513.

15) Gungorduk K, Yildirim G, Ark C. Is routine cervical dilatation necessary during elective caesarean section? A random- ised controlled trial. Aust N Z J Obstet Gynaecol.2009;49:263-267.

16) Ezegwui HU, Ogbuefi FC. Routine cervical dilatation during elective caesarean section. Should we continue? J Obstet Gynaecol. 2015;35:150-2.

17.Bollapragada SS, LC Edozien: Apparent absence of lochia after elective Caesarean section. J ObstetGynaecol.2002; 22:558.

18.Yancey MK, Clark P, Duff P. The frequency of glove contamination during cesarean delivery. Obstet Gynecol.1994; 83:538-542.

19. Duff P. Pathophysiology and management of postcesarean endomyometritis. ObstetGynecol.1986; 67:269-276. 